\title{
Über die Differenz zwischen theoretischer und empirischer Verteilungsfunktion
}

\author{
Von \\ WALTER NwF
}

\section{Einleitung}

Sei $F(x)$ die Verteilungsfunktion einer zufälligen Variablen $x$ und $S_{n}(x)$ die empirische Verteilungsfunktion einer dazugehörigen Stichprobe vom Umfang $n$, d.h.

$$
S_{n}(x)=\frac{1}{n} \sum_{k=1}^{n} u\left(x-x_{k}\right)
$$

wo $x_{1}, \ldots, x_{n}$ die Elemente der Stichprobe sind und $u(x)=0$ ist für $x<0$ und $u(x)=1$ für $x \geqq 0$. Im Jahre 1939 hat $\mathrm{N}$. SMrRvow [11] eine Arbeit veröffentlicht, in der die Anzahl der Werte von $x$ betrachtet wird, für welche die Differenz $S_{n}(x)-F(x)$ einen bestimmten Wert hat. Das wichtigste Resultat dieser Arbeit ist die Grenzverteilung dieser Anzahl für $n \rightarrow \infty \star$. Während also die Grenzverteilung schon längere Zeit bekannt ist, sind meines Wissens die exakten Verteilungen für endlichen Stichprobenumfang bisher nirgends angegeben worden. Sie herzuleiten ist der Zweck dieser Arbeit. Dabei beschränken wir uns allerdings auf solche Werte der Differenz $S_{n}(x)-F(x)$, die von der Form $r / n$ mit ganzzahligem $r$ sind. (Diese Beschränkung spielt insbesondere für den Grenzübergang $n \rightarrow \infty$ keine Rolle.) Bezeichnen wir die Anzahl der Werte von $x$, für die $S_{n}(x)-F(x)=r / n$ ist, mit $Z_{n}(r)$, so ist also die Verteilung dieser zufälligen Größe $Z_{n}(r)$ gesucht. Sie ist in den Formeln (27) und (29) enthalten.

Sodann wird gezeigt, wie die Smirnowsche Grenzverteilung aus der Verteilung für endliches $n$ durch Grenzübergang gewonnen werden kann, wodurch man einen neuen und elementaren Zugang zu den Resultaten von SMIRNow erhält.

Schließlich wird die Verteilung einer Größe $E_{n}$ angegeben, die in enger Beziehung zu der von KoLMOGORow eingeführten Größe

$$
D_{n}^{+}=\sup _{x}\left[S_{n}(x)-F(x)\right]
$$

steht und diese für große $n$ ersetzen kann.

Die Formulierungen der Arbeit beziehen sich auf den Fall einer stetigen Verteilungsfunktion $F(x)$. Am Ende von Abschnitt 2 wird jedoch gezeigt, daß die Resultate bei geeigneter Interpretation auch für den nichtstetigen Fall gültig sind.

* Surranow beschränkt sich auf stetige $F(x)$. Für nichtstetige $F(x)$ ist der Problemkreis der Sätze von Kolmogorow und Smirnow von P. Schmid [13] und H. Carnal [14] behandelt worden. 


\section{Hilfsformeln}

Dem Lehrbuch der Kombinatorik von E. NETTo [15] entnimmt man die folgende Beziehung

$$
\begin{aligned}
& A(j, s, a)=\sum_{k=0}^{j}\left(\begin{array}{l}
j \\
k
\end{array}\right)(-1)^{k}(k+a)^{s}=0 \\
& (j=1,2,3, \ldots ; s=0,1, \ldots, j-1 ; a \text { reell })
\end{aligned}
$$

(Seite 249, Formel (16)). Daraus folgt weiter

$$
B(n, a, b)=\sum_{k=0}^{n}\left(\begin{array}{l}
n \\
k
\end{array}\right)(k+a)^{k-1}(n+b-k)^{n-k}=\frac{(n+a+b)^{n}}{a}
$$

für $n \geqq 0, a \neq 0$ und beliebiges reelles $b$ (vgl. [15], Seite 249, letzte Formel). Durch binomische Entwicklung von $(n+b-k)^{n-k}=[(n+a+b)-(k+a)]^{n-k}$ erhält man nämlich mit (2) und wegen $A(0,-1, a)=1 / a$

$$
B(n, a, b)=\sum_{j=0}^{n}\left(\begin{array}{l}
n \\
j
\end{array}\right)(-1)^{j}(n+a+b)^{n-j} A(j, j-1, a)=\frac{(n+a+b)^{n}}{a} .
$$

Aus (3) folgt weiter

$$
C(n, b)==\sum_{k=1}^{n}\left(\begin{array}{l}
n \\
k
\end{array}\right) k^{k-1}(n+b-k)^{n-k}=n(n+b)^{n-1}
$$

für $n \geqq 0$ und beliebiges reelles $b$. Es ist nämlich

$$
C(n, b)=\lim _{a \rightarrow 0}\left(B(n, a, b)-\frac{(n+b)^{n}}{a}\right)=n(n+b)^{n-1} .
$$

\section{2. $r$-Werte}

2.1. Sei $F(x)$ stetig und vorerst streng monoton und $r$ eine ganze rationale Zahl. Ein Wert $x_{0}$ der zufälligen Variablen $x$ wird für eine bestimmte Stichprobe als $r$-Wert bezeichnet, wenn

$$
S_{n}\left(x_{0}\right)-F\left(x_{0}\right)=r / n
$$

ist (betr. nichtstetige $F^{\prime}(x)$ vgl. den Schluß dieses 2. Teils). Gegenstand der Untersuchung ist die Anzahl $Z_{n}(r)$ der $r$-Werte.

Für $r=0$ sollen im Sinne einer Konvention $x= \pm \infty$ als 0 -Werte betrachtet werden.

Ein $r$-Wert ist immer einer der Werte $x=z_{k}(0 \leqq k \leqq n)$, für die $F\left(z_{k}\right)=k / n$ ist. (Dabei ist $z_{0}=-\infty, z_{n}=+\infty$.)

Ist nun $F(x)$ nicht streng monoton und $F(x)=k / n$ längs einem Intervall, so setzen wir $z_{k}$ gleich dem Supremum dieses Intervalls (dies soll auch für $z_{0}$ gelten). Wenn wir von einem $r$-Wert verlangen, daß er immer gleich einem der Werte $z_{0}, \ldots, z_{n}$ sein soll, so wird der Fall einer nicht streng monotonen Funktion $F(x)$ ohne weiteres miterfaßt. 
Aus Symmetriegründen können wir uns im folgenden auf den Fall $r \geqq 0$ beschränken. Für $r>0$ kann ein $r$-Wert frühestens bei $z_{1}$ und spätestens bei $z_{n-r}$ auftreten.

Das Ereignis

$$
S_{n}(r, k)=\left\{z_{k} \text { ist ein } r \text {-Wert }\right\}
$$

tritt genau dann ein, wenn $(k+r)$ Elemente der Stichprobe $\leqq z_{k}$ sind. Also ist

$$
p\left(S_{n}(r, k)\right)=\left(\begin{array}{c}
n \\
k+r
\end{array}\right) \frac{k^{k+r}(n-k)^{n-k-r}}{n^{n}} .
$$

2.2. Für spätere Überlegungen ist es nützlich, den Stichproben vom Umfang $n$ das folgende ,Kugelmodell“" zuzuordnen:

$n$ Schachteln werden von 1 bis $n$ numeriert; sie entsprechen den Intervallen $\left(z_{0}, z_{1}\right], \ldots\left(z_{n-1}, z_{n}\right]$. Den Elementen der Stichprobe sollen $n$ Kugeln entsprechen, die zufallsmäßig und voneinander unabhängig in die $n$ Schachteln geworfen werden, wobei für jede Kugel jeder Schachtel die Wahrscheinlichkeit 1/n zukommen möge. Jeder Stichprobe $\sigma$ vom Umfang $n$ entspricht dann eine Verteilung $\varphi(\sigma)$ der $n$ Kugeln auf die $n$ Schachteln. Einer Menge (Ereignis) $S$ von Stichproben entspricht die Bildmenge $\varphi(S)$. Wir nennen $S$ vollständig, wenn aus

$$
\sigma=\left\{x_{1}, \ldots, x_{n}\right\} \in S \quad \text { folgt, daß auch } \sigma^{1}=\left\{x_{1}^{1}, \ldots, x_{n}^{1}\right\} \in S
$$

ist, falls für $i=1, \ldots, n x_{i}$ und $x_{i}^{1}$ im gleichen der Intervalle $\left(z_{k}, z_{k+1}\right]$ liegen (oder: falls $\varphi(\sigma)=\varphi\left(\sigma^{1}\right)$ ist). Man sieht dann leicht, daß für ein vollständiges Ereignis $S$ gilt: $p(S)=p(\varphi(S))$. Da es für alle in dieser Arbeit behandelten Fragestellungen nur darauf ankommt, wieviele Elemente der Stichprobe in jedem der Intervalle $\left(z_{k}, z_{k+1}\right]$ liegen, sind alle uns interessierenden Ereignisse vollständig, so daß ihre Wahrscheinlichkeiten im Kugelmodell bestimmt werden können.

Insbesondere ist $\varphi\left(S_{n}(r, k)\right)$ die Menge derjenigen Verteilungen der $n$ Kugeln, für die in den Schachteln Nr. 1 bis $k$ genau $(k+r)$ Kugeln liegen.

Aus der Existenz dieses Kugelmodells folgt übrigens, daß die Verteilung von $Z_{n}(r)$ von $F(x)$ unabhängig ist.

2.3. Wie schon erwähnt wurde, ist die vorliegende Arbeit für den Fall formuliert, daß $F(x)$ stetig ist. Die Resultate sind aber auch bei nichtstetigem $F(x)$ gültig, wenn der Begriff des $r$-Punktes etwas anders definiert wird. Da alles Folgende auf (7) beruht, ist die Definition so $\mathrm{zu}$ fassen, daß diese Gleichung wieder gilt.

Dazu führen wir vorerst die folgende zufällige Funktion $F^{*}(x)$ ein:

$$
F^{*}(x)=\text { gleichverteilt } z \text { wischen } F(x-0) \text { und } F(x+0)
$$

(insbesondere ist also $F^{*}(x)=F(x)$ mit Wahrscheinlichkeit 1, wenn $F(x)$ bei $x$ stetig ist).

Wir bezeichnen nun $x=x_{0}$ für eine bestimmte Stichprobe als $(r, k)$-Wert, wenn

1. $F\left(x_{0}-0\right) \leqq k / n \leqq F\left(x_{0}+0\right)$ und

2. für genau $(r+k)$ Elemente $x_{i}$ der Stichprobe $F^{*}\left(x_{i}\right) \leqq k / n$ ist. (Für nicht streng monotones $F(x)$ ist dieselbe Bemerkung zu machen wir unter 2.1.) 
Offensichtlich kann eine Unstetigkeitsstelle $x_{0}$ von $F(x)$ für verschiedene Werte von $r$ und $k$ ein $(r, k)$-Wert sein.

Man sieht ohne weiteres, daß bei stetigem $F(x)$ genau dann $x_{0}$ ein $(r, k)$-Wert ist, wenn $x_{0}=z_{k}$ und zugleich ein $r$-Wert ist.

Die Wahrscheinlichkeit dafür, daß für ein Element $x$ der Stichprobe $F^{*}(x) \leqq$ $\leqq k / n$ ist, ist nun gleich $k / n$. Die Wahrscheinlichkeit dafür, daß $x_{0}$, falls 1 . erfüllt ist, ein $(r, k)$-Wert ist, ist also wieder durch (7) gegeben. Setzt man nun $Z_{n}(r)$ gleich der Anzahl aller $(r, k)$-Werte für $k=0, \ldots, n$, so gelten unsere Resultate auch für nichtstetige $F(x)$.

\section{Der Fall $r=0$}

Die 0-Punkte entsprechen den Schnittpunkten der Graphen von $F(x)$ und $S_{n}(x)$. Aus (7) wird jetzt

$$
p\left(S_{n}(0, k)\right)=\left(\begin{array}{l}
n \\
k
\end{array}\right) \frac{k^{k}(n-k)^{n-k}}{n^{n}} .
$$

Wir betrachten nun das Ereignis

$$
T_{n}(s, k)=\left\{z_{k} \text { ist der } s \text {-te 0-Punkt (von links gezählt) }\right\} \text {. }
$$

Einer Stichprobe, die zu $T_{n}(s, k)$ gehört, entspricht im Kugelmodell eine Verteilung der Kugeln von der folgenden Art:

1. In den Schachteln Nr. 1 bis $k$ liegen $k$ Kugeln.

2. Diese $k$ Kugeln repräsentieren eine Stichprobe vom Umfang $k$, da für jede dieser Kugeln jeder Schachtel die (bedingte) Wahrscheinlichkeit $1 / k$ zukommt. Diese Stichprobe vom Umfang $k$ besitzt $s$ 0-Punkte.

Also wird

$$
p\left(T_{n}(s, k)\right)=p\left(S_{n}(0, k)\right) \cdot p\left(Z_{k}(0)=s\right) .
$$

Wenn wir im folgenden $Z_{n}$ anstelle von $Z_{n}(0)$ schreiben, so gilt für das Ereignis $Z_{n} \geqq s$ offensichtlich einerseits

$$
p\left(Z_{n} \geqq s\right)=\sum_{k=s}^{n+1} p\left(Z_{n}=k\right)
$$

und anderseits

$$
p\left(Z_{n} \geqq s\right)=\sum_{k=s-1}^{n} p\left(T_{n}(s, k)\right)=\sum_{k=s-1}^{n}\left(\begin{array}{l}
n \\
k
\end{array}\right) \frac{k^{k}(n-k)^{n-k}}{n^{n}} p\left(Z_{k}=s\right) .
$$

Aus (11) und (12) folgt

$$
\sum_{k=s}^{n+1} p\left(Z_{n}=k\right)=\sum_{k=8-1}^{n}\left(\begin{array}{l}
n \\
k
\end{array}\right) \frac{k^{k}(n-k)^{n-k}}{n^{n}} p\left(Z_{k}=s\right)
$$

und außerdem ist

$$
\sum_{k=2}^{n+1} p\left(Z_{n}=k\right)=1, \quad \text { insbesondere } \quad p\left(Z_{1}=2\right)=1 .
$$

Denn jede Stichprobe vom Umfang $n$ hat 2 oder $3 \ldots$ oder $(n+1) 0$-Punkte. 
Die Gleichungen (13) und (14) bilden ein lineares Gleichungssystem für die Größen $p\left(Z_{n}=s\right)$, durch das diese eindeutig bestimmt sind. Denn sie können aus (13) and (14) in der Reihenfolge $(n, s)=(1,2) ;(2,3),(2,2) ;(3,4),(3,3)$, $(3,2) ;(4,5), \ldots$ rekursiv berechnet werden.

Satz 1. Für $n=1,2,3, \ldots, s=2,3, \ldots, n+1$ ist

$$
\begin{aligned}
& p\left(Z_{n}=s\right)=\frac{(s-1)(n-1) !}{n^{s-1}(n-s+1) !}=\frac{n !}{n^{n}\left[\frac{n^{n-s+1}}{(n-s+1) !}-\frac{n^{n-s}}{(n-s) !}\right]} \\
& p\left(Z_{n} \geqq s\right)=\frac{n !}{n^{n}} \frac{n^{n-s+1}}{(n-s+1) !} .
\end{aligned}
$$

Beweis. Vorerst folgt (16) ohne weiteres aus der zweiten Darstellung in (15). Um (15) zu beweisen, genügt nach dem Vorhergehenden der Nachweis, daß die Gleichungen (13) und (14) mit (15) erfüllt sind.

Was (14) betrifft, so folgt dies unmittelbar aus der 2. Darstellung in (15).

Setzt man (15) auf der rechten Seite von (13) ein, so erhält man nach kurzer Umformung mit der Substitution $i=k-s+1$ den Ausdruck

$$
\frac{n !(s-1)}{(n-s+1) ! n^{n}} \sum_{i=0}^{n-s+1}\left(\begin{array}{c}
n-s+1 \\
i
\end{array}\right)(i+s-1)^{i-1}(n-i-s+1)^{n-i-s+1}
$$

der nach (3) gleich

$$
\frac{n !}{n^{n}} \frac{n^{n-s+1}}{(n-s+1) !},
$$

also nach (16) gleich der linken Seite von (13) ist.

\section{Ein Hilisproblem}

Im Anschluß an das Kugelmodell (s. Abschnitt 2) lösen wir die folgende Aufgabe:

Gegeben sind $n$ Schachteln und $n-r$ Kugeln $(0 \leqq r \leqq n)$. Wieder komme jeder Schachtel für jede Kugel die Wahrscheinlichkeit $1 / n$ zu und die Nummern der Schachteln, die den verschiedenen Kugeln zugeordnet sind, seien unabhängig. Wir suchen $p(P(r, n))$ für das Ereignis

$$
\begin{aligned}
& P(r, n)=\{\text { für kein } k(k=1, \ldots, n-r) \text { befinden sich in den Schach- } \\
& \text { teln Nr. } 1 \text { bis } k \text { genau } k \text { Kugeln }\} \text {. }
\end{aligned}
$$

Das komplementäre Ereignis ist

$$
\bar{P}(r, n)=\bigcup_{k=1}^{n-r} Q(k, r, n)
$$

wo

Dann gilt

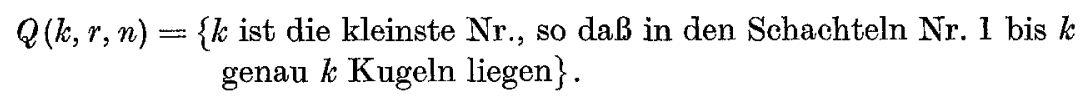

$$
p(Q(k, r, n))=\left(\begin{array}{c}
n-r \\
k
\end{array}\right) \frac{k^{k}(n-k)^{n-r-k}}{n^{n-r}} \cdot \frac{1}{k} .
$$

* Für $s=(n+1)$ ist im 2. Ausdruck der 2. Summand in der Klammer wegzulassen! 
Ohne den Faktor $1 / k$ ist dies nämlich die Wahrscheinlichkeit dafür, daß in den ersten $k$ Schachteln $k$ Kugeln liegen. Der Faktor $1 / k$ ist dann die (bedingte) Wahrscheinlichkeit dafür, daß kein kleineres $k$ mit dieser Eigenschaft existiert. Wenn dies nämlich der Fall ist, so entsprechen die in den Schachteln Nr. 1 bis $k$ liegenden Kugeln einer Stichprobe vom Umfang $k$, die nur die minimale Zahl von 20 -Punkten besitzt. Nach (15) ist aber $p\left(Z_{k}=2\right)=1 / k$.

Da die Ereignisse $Q(k, r, n)$ für verschiedene Werte von $k$ disjunkt sind, folgt aus (18)

$$
p(\bar{P}(r, n))=\sum_{k=1}^{n-r} p(Q(k, r, n))=\frac{1}{n^{n-r}} \sum_{k=1}^{n-r}\left(\begin{array}{c}
n-r \\
k
\end{array}\right) k^{k-1}(n-k)^{n-r-k}=1-\frac{r}{n}
$$

nach (4). Also ist

$$
p(P(r, n))=r / n .
$$

\section{Die Verteilung von $Z_{n}(r)$ für $r \geqq 1$}

5.1. Wir berechnen zuerst $p\left(Z_{n}(r) \geqq 1\right)$.

Es gilt

$$
\left(Z_{n}(r) \geqq 1\right)=\bigcup_{k=r}^{n-1} L_{n}(r, k)
$$

mit

$L_{n}(r, k)=\left\{\right.$ es existiert mindestens ein $r$-Punkt und der letzte ist $\left.z_{n-k}\right\}$. Da die $L_{n}(r, k)$ für verschiedene $k$ disjunkt sind, ist

$$
p\left(Z_{n}(r) \geqq 1\right)=\sum_{k=r}^{n-1} p\left(L_{n}(r, k)\right) .
$$

$L_{n}(r, k)$ tritt genau dann ein, wenn in den letzten $k$ Intervallen $\left(z_{n-k}, z_{n-k+1}\right]$, $\ldots,\left(z_{n-1}, z_{n}\right]$ genau $(k-r)$ Elemente der Stichprobe liegen und für kein $l>0$ gilt, daß in den ersten $l$ von diesen Intervallen genau $l$ Elemente liegen. Auf Grund des Hilfsproblems (Abschnitt 4), in dem wir nur $n$ durch $k$ zu ersetzen haben, folgt also

$$
\begin{aligned}
p\left(Z_{n}(r)\right. & \geqq 1)=\sum_{k=r}^{n-1}\left(\begin{array}{c}
n \\
k-r
\end{array}\right) \frac{k^{k-r}(n-k)^{n-k+r}}{n^{n}} p(P(r, k)) \\
& =\frac{r}{n^{n}} \sum_{k=r}^{n-1}\left(\begin{array}{c}
n \\
k-r
\end{array}\right) k^{k-r-1}(n-k)^{n-k+r} .
\end{aligned}
$$

5.2. Wir gehen zur Berechnung von $p\left(Z_{n}(r) \geqq s\right)$ über, wobei $s \geqq 2$ sei. Wenn $Z_{n}(r) \geqq s$ ist, so sei $z_{k_{1}}$ der größte $r$-Wert. $z_{k_{0}}$ sei der $s$-te $r$-Wert, von rechts gezählt. Im Kugelmodell entspricht dann dieser Stichprobe die folgende Situation:

In den ersten $k_{0}$ Schachteln befinden sich $\left(k_{0}+r\right)$ Kugeln, in den letzten $\left(n-k_{1}\right)$ Schachteln $\left(n-k_{1}-r\right)$ Kugeln. In den mittleren $l=k_{1}-k_{0}>0$ Schachteln sind $l$ Kugeln. Diese $l$ Kugeln sind auf die $l$ Schachteln so verteilt, daß die entsprechende Stichprobe vom Umfang $l$ genau $s$ 0-Werte enthält (entsprechend den $s$ letzten $r$-Werten der ursprünglichen Stichprobe). 
Den $(n-l)$ Kugeln, die sich in den $(n-l)$ äußeren Schachteln befinden, entspricht eine Stichprobe vom Umfang $(n-l)$, die mindestens einen $r$-Wert besitzt. Da $l$ von $s-1$ bis $n-r+1$ variieren kann, folgt

$$
p\left(Z_{n}(r) \geqq s\right)=\sum_{l=s-1}^{n-r+1}\left(\begin{array}{c}
n \\
l
\end{array}\right) \frac{l^{l}(n-l)^{n-l}}{n^{n}} p\left(Z_{1}(0) \geqq s\right) p\left(Z_{n-l}(r) \geqq 1\right) .
$$

Mit Hilfe von (16) und (23) folgt nach einigen Zwischenrechnungen, und indem der Index $k$ aus (23) ersetzt wird durch $\mu=n-l+r-k$, sodann der Index $l$ durch $k=l-s+1$

$$
=\frac{r(s-1) n !}{(n-s+1) ! n^{n}} \sum_{\mu=r+1}^{n-s+1}\left(\begin{array}{c}
n-s+1 \\
\mu
\end{array}\right)(\mu-r)^{\mu} \sum_{k=0}^{m}\left(\begin{array}{c}
m \\
k
\end{array}\right)(k+s-1)^{k-1}(m+r-k)^{m-k-1}
$$

mit $m=n-\mu-s+1$.

Die innere Summe wird

$$
X=X_{1}+X_{2}
$$

mit $\quad X_{1}=\sum_{k=0}^{m-1}(m-1)(k+s-1)^{k-1}(m+r-k)^{m-k-1}$

$$
=\frac{(n+r-\mu)^{n-s-\mu}}{s-1} \operatorname{nach}(3)
$$

und $\quad X_{2}=\sum_{k=1}^{m}\left(\begin{array}{c}m-1 \\ k-1\end{array}\right)(k+s-1)^{k-1}(m+r-k)^{m-k-1}$

$$
=\frac{(n+r-\mu)^{n-s-\mu}}{r} \text { ebenfalls nach (3). }
$$

Nach (25) ist also

$$
X=\frac{r+s-1}{r(s-1)}(n+r-\mu)^{n-s-\mu} .
$$

Aus (24) folgt jetzt

Satz 2. Für $n \geqq 1, r \geqq 0, s \geqq 1$ ist

$$
p\left(Z_{n}(r) \geqq s\right)=\frac{(r+s-1) n !}{(n-s+1) ! n^{n}} \sum_{\mu=r+1}^{n-s+1}\left(\begin{array}{c}
n-s+1 \\
\mu
\end{array}\right)(\mu-r)^{\mu}(n+r-\mu)^{n-s-\mu} .
$$

Bisher ist dies allerdings nur für $r \geqq 1$ und $s \geqq 2$ bewiesen.

Daß (27) auch für $r=0$ gilt, sieht man an der mit (27) äquivalenten Darstellung (29) durch Vergleich mit (16).

Setzt man ferner in (27) $s=1$ und $\mu=n+r-k$, so geht (27) über in (23) und gilt also auch für $s=1$.

Formel (27) ist für die numerische Berechnung unbequem, wenn $n$ groß, $r$ und $s$ jedoch klein sind. Für diesen Fall erhält man eine bessere Darstellung wie folgt: 
Durch die Substitution $k=n-s+1-\mu$ erhält man

$$
\begin{gathered}
\sum_{\mu=0}^{n-s+1}\left(\begin{array}{c}
n-s+1 \\
\mu
\end{array}\right)(\mu-r)^{\mu}(n+r-\mu)^{n-s-\mu} \\
=\sum_{k=0}^{n-s+1}\left(\begin{array}{c}
n-s+1 \\
k
\end{array}\right)(k+r+s-1)^{k-1}(n-r-s+1-k)^{n-s+1-k}=\frac{n^{n-s+1}}{r+s-1}
\end{gathered}
$$

nach (3).

Also wird mit (27)

$$
\begin{aligned}
p\left(Z_{n}(r) \geqq s\right)= & \frac{n !}{(n-s+1) ! n^{n}}\left[n^{n-s+1}-(r+s-1) \times\right. \\
& \times \sum_{\mu=0}^{r-1}\left(\begin{array}{c}
n-s+1 \\
\mu
\end{array}\right)(\mu-r)^{\mu}(n+r-\mu)^{n-s-\mu]}
\end{aligned}
$$

\section{Die Grenzverteilung von N. Smirnow}

Aus (27) läßt sich die Grenzverteilung von N. SurRnow gewinnen. Wir beschränken uns in dieser Arbeit, um nicht zu lang zu werden, auf eine Skizze des Beweises.

Wir setzen $r=\varrho \sqrt{n}, s=\sigma \sqrt{n}$, wo $\varrho, \sigma$ konstant sind. Ersetzt man noch den Summationsindex $\mu$ durch $t=\mu / n$, so erhält man für den einzelnen Summanden $S_{\mu}$ in (27) unter Verwendung der Stirlingschen Näherung nach einigen Rechnungen

$$
\lim _{n \rightarrow \infty}\left(n S_{\mu}\right)=\frac{\varrho+\sigma}{\sqrt{2 \pi}} \frac{1}{\sqrt{t(1-t)^{3}}} \exp \left[-\frac{1}{2}\left(\frac{\varrho^{2}}{t}+\frac{(\varrho+\sigma)^{2}}{1-t}\right)\right] .
$$

Da $\mu$ in (27) in Schritten von 1 von $r$ bis $n-s+1$ läuft, läuft $t$ in Schritten von $\frac{1}{n}$ von $\frac{\varrho}{\sqrt{n}}$ bis $1-\frac{\sigma}{\sqrt{n}}+\frac{1}{n}$. Somit wird (wir verzichten auf die genaue Durchführung des Grenzüberganges):

$$
\begin{aligned}
\lim _{n \rightarrow \infty} p\left(Z_{n}(\varrho \sqrt{n})\right. & \left.\geqq \sigma V^{\sqrt{n}}\right)= \\
& =\frac{\varrho+\sigma}{\sqrt{2 \pi}} \int_{0}^{1} \frac{d t}{\sqrt{t(1-t)^{3}}} \exp \left[-\frac{1}{2}\left(\frac{\varrho^{2}}{t}+\frac{(\varrho+\sigma)^{2}}{1-t}\right)\right]=\mathrm{e}^{-1 / 2(2 \varrho+\sigma)^{2}}
\end{aligned}
$$

entsprechend dem. Resultat von SmiRnow*.

\section{Die Größe $D_{n}^{+}$von Kolmogorow}

Die zufällige Größe

$$
D_{n}^{+}=\sup _{k}\left[S_{n}(x)-F(x)\right]
$$

ist von KoLmogorow [8] untersucht worden, der ihre asymptotische Verteilung für $n \rightarrow \infty$ angegeben hat. Wir wollen die Verteilung von $D_{n}^{++}$für endlichen Stich-

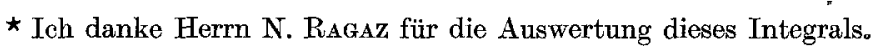


probenumfang $n$ ableiten, wobei wir jedoch die Definition so modifizieren, daß nur die Werte $x=z_{k}(0 \leqq k \leqq n)$ berücksichtigt werden. Wir setzen also

$$
E_{n}^{+}=\sup _{k}\left[S_{n}\left(z_{k}\right)-F\left(\bar{z}_{k}\right)\right] \text {. }
$$

Offensichtlich ist $E_{n}^{+} \leqq D_{n}^{+}$. Die Differenz der beiden Größen geht jedoch gegen Null für $n \rightarrow \infty$, so daß das asymptotische Verhalten in beiden Fällen dasselbe ist.

Nun ist offensichtlich $E_{n}^{+} \geqq r / n$ genau dann, wenn $Z_{n}(r) \geqq 1$ ist. Also folgt mit (23)

$$
p\left(E_{n}^{+} \geqq r\right)=\frac{r}{n^{n}} \sum_{k=r}^{n-1}\left(\begin{array}{c}
n \\
k-r
\end{array}\right) k^{k-r-1}(n-k)^{n-k+r} .
$$

Andere Ausdrücke für diese Verteilung erhält man aus (27) und (29). Durch Grenzübergang kann aus (30) die Grenzverteilung von $D_{n}^{+}$für $n \rightarrow \infty$ gefunden werden.

Die ebenfalls von Kolmogorow eingeführte Größe $D_{n}=\sup _{x}\left|S_{n}(x)-F(x)\right|$ kann mit dem in dieser Arbeit verwendeten Formalismus entsprechend behandelt werden, was jedoch einer besonderen Arbeit vorbehalten sei.

\section{Literatur}

[1] Anderson, T. W., and D. A. Darling: Asymptotic theory of certain "goodness of fit" criteria based on stochastic processes. Ann. math. Statistics 23, 193-212 (1952).

[2] Donsker, M. O.: Justification and extension of Doob's heuristic approach to the Kolmogoroff-Smirnow theorems. Ann. math. Statistics 23 277-281 (1952).

[3] Doos, L.: Heuristic approach to the Kolmogoroff-Smirnow theorems. Ann. math. Statistics 20, 393-403 (1949).

[4] FeLLer, W.: On the Kolmogoroff-Smirnow limit theorems for empirical distributions. Ann. math. Statistics 19, 177-189 (1948).

[5] Gliwenko, W.: Sulla determinazione empirica delle leggi di probabilita. Giorn. Ist. Ital. Attuari 4, 92-99 (1933).

[6] GNedenko, B., und W. Korol JUK: Utber die maximale Divergenz von zwei empirischen Verteilungen. Doklady Akad. Nauk SSSR, n. Ser. 80, 525-528 (1951).

[7] -- Kontrolle der Unveränderlichkeit der Wahrscheinlichkeitsverteilung in zwei voneinander unabhängigen Stichproben. Math. Nachr. 12, 29-63 (1954).

[8] Kolmogorofr, A.: Sulla determinazione empirica di una legge di distribuzione. Giorn. Ist. Ital. Attuari 4, 83-91 (1933).

[9] vor MIsEs, R.: Wahrscheinlichkeitsrechnung und ihre Anwendung in der Statistik und theoretischen Physik (1.931), S. $316 \mathrm{ff}$.

[10] Sminnow, N.: Sur la distribution de $\omega^{2}$. C. R. Acad. Sci. 220, 449-452 (1936).

[11] - Sur les écarts de la courbe de distribution empirique. Rec. math. Moseou, N. s. 6, $3-24$ (1939).

[12] - Abschätzung der Divergenz zwischen den empirischen Verteilungskurven in zwei unabhängigen Stichproben. Bull. Math. Univ. Moscou, Sér. internat. 2, Fasc. 2, $1-16(1939)$.

[13] ScHmrn, P.: On the Kolmogoroff and Smirnow theorems for discontinuous distribution functions. Ann. math. Statistics 29, 1011-1027 (1958).

[14] CARnal, H.: Sur les théorèmes de Kolmogoroff et Smirnow dans le cas d'une distribution discontinue. Commentarii math. Helvet. 37, 19-35 (1962).

[15] Netro, E.: Lehrbuch der Combinatorik. Leipzig: B. G. Teubner 1927.

Institut für Angewandte Mathematik der Universität, Bern (Schweiz) 